\title{
Pulsed Triple Frequency Modulation for Frequency Stabilization and Control of Two Lasers to an Optical Cavity
}

\author{
William Williams \\ Smith College,wwilliams@smith.edu \\ Maria Teresa Herd \\ Assumption College \\ E. C. Cook \\ Smith College
}

Follow this and additional works at: https://scholarworks.smith.edu/phy_facpubs

Part of the Physics Commons

\author{
Recommended Citation \\ Williams, William; Herd, Maria Teresa; and Cook, E. C., "Pulsed Triple Frequency Modulation for Frequency \\ Stabilization and Control of Two Lasers to an Optical Cavity" (2020). Physics: Faculty Publications, Smith \\ College, Northampton, MA. \\ https://scholarworks.smith.edu/phy_facpubs/77
}

This Article has been accepted for inclusion in Physics: Faculty Publications by an authorized administrator of Smith ScholarWorks. For more information, please contact scholarworks@smith.edu 


\section{Pulsed triple frequency modulation for frequency stabilization and control of two lasers to an optical cavity}

Cite as: Rev. Sci. Instrum. 91, 085116 (2020); https://doi.org/10.1063/5.0010085

Submitted: 06 April 2020 . Accepted: 09 August 2020 . Published Online: 21 August 2020

(D) W. D. Williams, (D) M. T. Herd, and (D) E. C. Cook

ErssMark

\section{ARTICLES YOU MAY BE INTERESTED IN}

An introduction to Pound-Drever-Hall laser frequency stabilization

American Journal of Physics 69, 79 (2001); https://doi.org/10.1119/1.1286663

Digital laser frequency and intensity stabilization based on the STEMlab platform (originally Red Pitaya)

Review of Scientific Instruments 91, 083001 (2020); https://doi.org/10.1063/5.0009524

Laser stabilization to neutral $\mathrm{Yb}$ in a discharge with polarization-enhanced frequency modulation spectroscopy

Review of Scientific Instruments 91, 123002 (2020); https://doi.org/10.1063/5.0019252

\section{Challenge us.}

What are your needs for periodic signal detection?

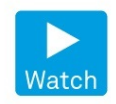

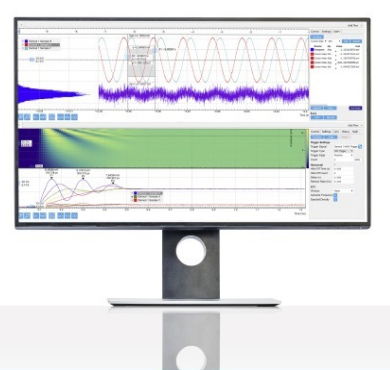




\title{
Pulsed triple frequency modulation for frequency stabilization and control of two lasers to an optical cavity
}

\author{
Cite as: Rev. Sci. Instrum. 91, 085116 (2020); doi: 10.1063/5.0010085 \\ Submitted: 6 April 2020 - Accepted: 9 August 2020 • \\ Published Online: 21 August 2020

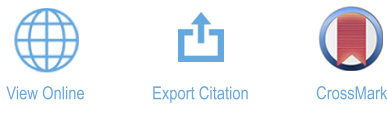

W. D. Williams, ${ }^{1, a)}$ (D) M. T. Herd, ${ }^{2}$ (D) and E. C. Cook ${ }^{1}$ (D)

AFFILIATIONS
${ }^{1}$ Physics Department, Smith College, Northampton, Massachusetts 01063, USA
${ }^{2}$ Department of Biological and Physical Sciences, Assumption College, Worcester, Massachusetts 01609, USA

a) Author to whom correspondence should be addressed: wwilliams@smith.edu

\begin{abstract}
We present a method to stabilize two lasers to an optical cavity using pulsed triple frequency modulation. The setup allows simultaneous Pound-Drever-Hall stabilization, as well as independent frequency control, while removing interference terms that limit the frequency scan range and allowing for smaller modulation depths. A review of single, dual, and triple frequency modulation is also presented in addition to a discussion of how to effectively turn pulsed triple frequency modulation into independent dual frequency modulation for each laser. This method would increase the scan range to half the free spectral range.
\end{abstract}

Published under license by AIP Publishing. https://doi.org/10.1063/5.0010085

\section{INTRODUCTION}

Stabilizing and controlling the frequency of lasers is critical to many modern physics experiments. The most common methods to stabilize the frequency of a laser use an atomic source or an ultra-low expansion (ULE) cavity. There are various methods for stabilizing a laser to an atomic source including saturated absorption, the dichroic atomic vapor laser lock (DAVLL), ${ }^{1}$ Sagnac interferometry, ${ }^{2}$ polarization spectroscopy, ${ }^{3}$ frequency modulation (FM) spectroscopy, ${ }^{4}$ and modulation transfer spectroscopy. ${ }^{5,6}$ Stabilizing the frequency of a laser to a ULE cavity typically uses the PoundDrever-Hall (PDH) technique. Given a stabilization method, many experiments then need to controllably scan the frequency of the laser with respect to a specific frequency reference point such as an atomic transition or an optical cavity mode. Common methods to accomplish this task include using a double-pass acousto-optical modulator (AOM), an offset/transfer lock, ${ }^{8}$ or an offset sideband lock. ${ }^{9}$

Dual frequency modulation, also known as a dual sideband lock, ${ }^{9}$ is a type of offset sideband lock that uses two successive electro-optical modulators (EOMs), which phase modulate the lasers and consequently generate sidebands. Alternative methods for offset sideband locks are also discussed by Thorpe et al., ${ }^{9}$ but for the purposes of this paper, we will only consider dual frequency modulation, which is the method used by our group for stabilizing and controlling the frequency of a single laser. ${ }^{10}$ To use dual frequency modulation, a laser first passes through a resonant "low"frequency EOM, driven at $f_{\text {low }}=\Omega_{\text {low }} / 2 \pi$. The laser then passes through a broadband EOM creating high-frequency sidebands at $f_{E O M}$. In the small modulation limit, there are a total of nine frequency components on the laser. Next, the light is coupled to the optical cavity with the reflection incident upon a photodiode in a typical Pound-Drever-Hall setup. The output signal from the photodiode is demodulated at $\Omega_{\text {low }}$ to produce error signals for stabilization. As a function of frequency, there are then three PDH error signals available for laser stabilization: one centered at the negative sideband of the high-frequency EOM, one centered at the carrier, and one centered at the positive sideband of the high-frequency EOM. If the laser is stabilized to the ULE cavity using the negative sideband of the high-frequency EOM, the frequency of the laser is given by

$$
f_{L}=f_{n}+f_{E O M},
$$

where $f_{L}$ is the frequency of the laser and $f_{n}=n c /(2 L)$ is the resonant frequency for the $n$th mode of the ULE cavity with effective 
length $L$ at $f_{n}$. By scanning $f_{E O M}$, the frequency of the laser is similarly scanned. A limitation of dual frequency modulation is that the laser cannot be scanned over certain EOM frequencies. For example, when $f_{E O M} \approx \frac{F S R}{2} \pm f_{\text {low }}$, the positive high-frequency sideband from the adjacent cavity mode begins to be transmitted through the cavity. When this happens, the error signal for the negative sideband is distorted, which interferes with the zero-crossing for the PDH lock. Despite this limitation, the scan range is typically larger than the scan range of a double-pass AOM setup, which is AOM bandwidth limited.

Triple frequency modulation is an extension of the dual frequency technique that allows simultaneous offset sideband stabilization of two lasers to a single ULE cavity. ${ }^{11}$ The principle is identical to dual frequency modulation, but the high-frequency EOM is driven by the combined signal from two function generators to allow stabilization of two lasers using independently tunable sidebands. As shown in Sec. II, there are additional high-order sidebands created by modulating the broadband EOM at two frequencies. This both removes power from the desired sidebands and limits the scan range. Phase interference effects also arise from relative phase or frequency drifts of the two function generators. This manuscript improves upon triple frequency modulation by alternately pulsing the modulation outputs, as described in Sec. II D. By alternating the modulation outputs, the number of sidebands is reduced, conserving laser power and increasing the available scan range. Furthermore, we discuss a method to implement pulsed triple frequency modulation so that the scan range is extended up to half the free spectral range, equivalent to dual frequency modulation.

This manuscript is organized as follows: Sec. II gives a mathematical overview of single, dual, and triple frequency modulation, Sec. II D discusses pulsed triple frequency modulation, Sec. III describes the experimental setup, Sec. IV presents the experimental results, and Sec. $V$ concludes the paper.

\section{BACKGROUND}

\section{A. Single frequency modulation}

Single frequency modulation requires one EOM. After passing through the EOM, the electric field of the laser beam has been phase modulated to have the form

$$
E=E_{0} e^{i(\omega t+\beta \sin (\Omega t))}=E_{0} e^{i \omega t} e^{i \beta \sin (\Omega t)},
$$

where $E_{0}$ is the (complex) electric field amplitude, $\omega$ is the angular frequency of the laser, $\Omega$ is the phase-modulation frequency, and $\beta$ is the modulation depth. The second expression can be expanded using the Jacobi-Anger expansion

$$
\begin{aligned}
e^{i \beta \sin (\Omega t)} & =\sum_{n=-\infty}^{\infty} J_{n}(\beta) e^{i n \Omega t} \\
& =J_{0}(\beta)+\sum_{\mathrm{n}=1}^{\infty}\left(J_{n}(\beta) e^{i n \Omega t}+(-1)^{n} J_{n}(\beta) e^{-i n \Omega t}\right) \\
& \approx J_{0}(\beta)+J_{1}(\beta) e^{i \Omega t}-J_{1}(\beta) e^{-i \Omega t},
\end{aligned}
$$

where Eq. (4) uses the Bessel identity $J_{-n}(\beta)=(-1)^{n} J_{n}(\beta)$ and Eq. (5) is the small-modulation-depth $(\beta \ll 1)$ approximation in which higher-order sidebands $(n \geq 2)$ are negligible. Combining Eqs. (2) and (4), we get

$$
\begin{aligned}
\frac{E}{E_{0}}= & J_{0}(\beta) e^{i \omega t} \\
& +\sum_{\mathrm{n}=1}^{\infty}\left(J_{n}(\beta) e^{i(\omega+n \Omega) t}+(-1)^{n} J_{n}(\beta) e^{i(\omega-n \Omega) t}\right) \\
\approx & J_{0}(\beta) e^{i \omega t}+J_{1}(\beta) e^{i(\omega+\Omega) t}-J_{1}(\beta) e^{i(\omega-\Omega) t} .
\end{aligned}
$$

Conceptually, in the small-modulation-depth limit, this can be thought of as three separate, copropagating laser beams with frequencies $\omega, \omega+\Omega$, and $\omega-\Omega$, respectively. The last two terms are referred to as the positive and negative sidebands. For the rest of the paper, we will use Eq. (7) with the subscript "low" to represent the electric field of the laser after leaving the first EOM. The modulation frequency for this experiment is $\Omega_{\text {low }} / 2 \pi=20 \mathrm{MHz}$ and is smaller than the high-frequency modulation described below.

\section{B. Dual frequency modulation}

Dual frequency modulation uses two EOMs. In the smallmodulation approximation, the electric field of the laser beam after the first EOM is described by Eq. (7). Driving the second EOM at $\Omega_{A}$, the electric field leaving the second EOM is given by

$$
\begin{aligned}
\frac{E}{E_{0}} \approx & \left(J_{0}\left(\beta_{\text {low }}\right) e^{i \omega t}+J_{1}\left(\beta_{\text {low }}\right) e^{i\left(\omega+\Omega_{\text {low }}\right) t}\right. \\
& \left.-J_{1}\left(\beta_{\text {low }}\right) e^{i\left(\omega-\Omega_{\text {low }}\right) t}\right) e^{i \beta_{A} \sin \left(\Omega_{A} t\right)} .
\end{aligned}
$$

We find that for dual frequency modulation, driving the second EOM in the small modulation limit produces large enough error signals for laser stabilization. ${ }^{10}$ In this case, Eq. (8) represents nine frequency terms on the laser beam (three error signals when the reflected light from the cavity is demodulated at $\Omega_{\text {low }}$ for PDH stabilization). Each frequency term has the form

$$
J_{|m|}\left(\beta_{\text {low }}\right) J_{|j|}\left(\beta_{A}\right) e^{i\left(\omega+m \Omega_{l o w}+j \Omega_{A}\right) t}
$$

where $m=-1,0$, or 1 and $j=-1,0,1$.

\section{Triple frequency modulation}

Triple frequency modulation, which can be used to independently stabilize and scan two laser beams, ${ }^{11}$ requires driving the second EOM at two frequencies, $\Omega_{A}$ and $\Omega_{B}$. The expression for triple frequency modulation is

$$
\begin{aligned}
\frac{E}{E_{0} \approx} & \left(J_{0}\left(\beta_{\text {low }}\right) e^{i \omega t}+J_{1}\left(\beta_{\text {low }}\right) e^{i\left(\omega+\Omega_{\text {low }}\right) t}\right. \\
& \left.-J_{1}\left(\beta_{\text {low }}\right) e^{i\left(\omega-\Omega_{\text {low }}\right) t}\right) e^{i\left(\beta_{A} \sin \left(\Omega_{A} t\right)+\beta_{B} \sin \left(\Omega_{B} t\right)\right)} .
\end{aligned}
$$

In order to create high-frequency sidebands of sufficient amplitude to stabilize a laser, we find that the second EOM must be driven outside the small-modulation limit and include second-order terms in the expansion of Eq. (10). Equation (10) then represents 75 frequency terms. Each frequency term has the form

$$
J_{|m|}\left(\beta_{\text {low }}\right) J_{|j|}\left(\beta_{A}\right) J_{|k|}\left(\beta_{B}\right) e^{i\left(\omega+m \Omega_{\text {low }}+j \Omega_{A}+k \Omega_{B}\right) t},
$$

where $m=-1,0$, or $1, j=-2,-1,0,1$, or 2 , and $k=-2,-1,0,1$, or 2 . After demodulation at $\Omega_{\text {low }}$, this results in $25 \mathrm{PDH}$ error signals on 
each laser beam. Due to the cyclical nature of optical cavities, all 25 error signals appear in one free spectral range scan, which limits the potential scan range of each laser. We will refer to any term where both $j$ or $k$ do not equal zero as interference terms.

If one of these error signals interferes with the error signal used to stabilize the laser, the lock point is shifted or the lock can be lost. For example, suppose $\Omega_{A} / 2 \pi=900 \mathrm{MHz}, \Omega_{B} / 2 \pi=1200 \mathrm{MHz}$, and the free spectral range of the optical cavity is $1500 \mathrm{MHz}$. We would like to stabilize laser 1 to the negative $900 \mathrm{MHz}$ sideband $(j=$ $-1, k=0)$ and laser 2 to the negative $1200 \mathrm{MHz}$ sideband $(j=0, k$ $=-1$ ). Concerning ourselves only with laser 1 , there are 25 possible error signals on this laser beam. From Eq. (11), there is a sideband at $2100 \mathrm{MHz}(j=1, k=1)$, two sidebands at $600 \mathrm{MHz}(j=-2, k=2$ and $j=2, k=-1)$, and one sideband at $-2400 \mathrm{MHz}(j=0, k=-2)$ that are also resonant with adjacent cavity modes. If we were to scan laser 1 around this lock point, there are five error signals interfering with one another making the lock unstable. In addition, there are five error signals at the lock point of laser 2 .

\section{Pulsed triple frequency modulation}

To eliminate many of the unwanted error signals, we amplitude-modulate the modulation depths of the second EOM with a square wave such that the modulation depth of $\Omega_{A}$ is $\beta_{A}$ ("on") and $\Omega_{B}$ is 0 ("off"), or $\Omega_{A}$ is 0 ("off") and $\Omega_{B}$ is $\beta_{B}$ ("on"). Further details on the timing and implementation of pulsed frequency modulation are given in Secs. III and IV. For reference, the timing of the modulation trigger is shown in Fig. 5.

In the first case, all frequency terms where $k \neq 0$ are eliminated $\left(J_{0}\left(\beta_{B}=0\right)=1\right.$, while $\left.J_{k \neq 0}\left(\beta_{B}=0\right)=0\right)$. Likewise, with $\beta_{B}$ "on" and $\beta_{A}=0$, all frequency terms where $j \neq 0$ are eliminated.

Since the interference terms are eliminated by pulsed frequency modulation, of the 75 frequency terms ( 25 error signals) represented by Eq. (11), only a total of 27 frequency terms (9 error signals) are present, although not at the same time. Due to the amplitude modulation on the modulation depths, only 15 frequency terms (five error signals) exist at any given time; the carrier error signal is the only error signal present at all times. Since the laser power is no longer divided simultaneously between 75 frequency components, the modulation depths of the high-frequency EOM can then be reduced while still maintaining sidebands of sufficient amplitude for laser stabilization. As a result, we find that for pulsed triple frequency modulation, both EOMs can be driven in the small-modulation limit. In this limit, the complexity is reduced back to only nine frequency terms (three error signals) present at a given time.

\section{IMPLEMENTATION}

Figure 1 shows the experimental setup for pulsed triple frequency modulation. For our setup, laser 1 is a frequency-doubled Ti-Sapphire laser operating at $470 \mathrm{~nm}$ and laser 2 is a Toptica Photonics external-cavity diode laser (ECDL) at $457 \mathrm{~nm}$. Both lasers are stabilized to a ULE cavity kept under vacuum at a pressure of $1 \times 10^{-7}$ Torr and temperature-stabilized to $0.01{ }^{\circ} \mathrm{C}$. ${ }^{12}$ The two lasers are combined using a dichromatic mirror (Semrock LM01466-25) and sent through a resonant $20 \mathrm{MHz}$ EOM (Photonics Technologies Ltd. EOM-01-20-U) followed by a temperature-stabilized,

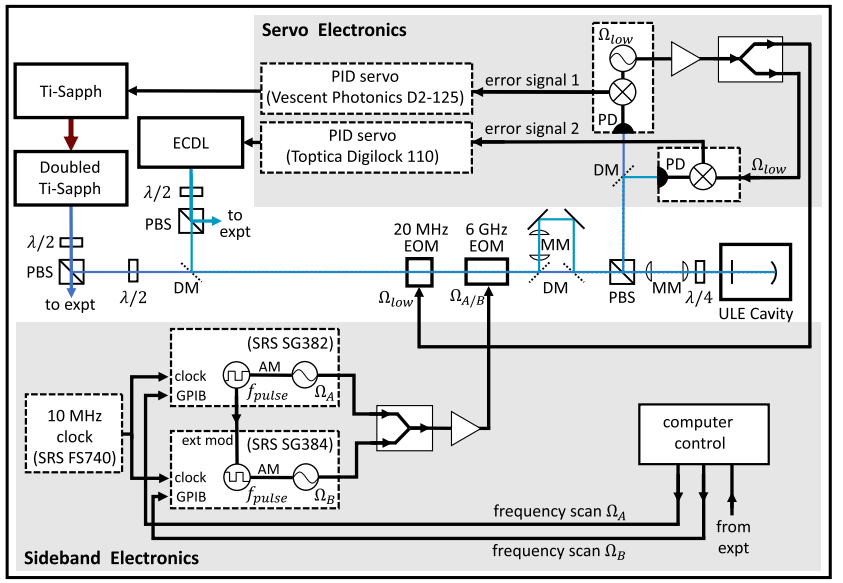

FIG. 1. A simplified experimental schematic for pulsed triple frequency modulation. See the text for details. The following abbreviations are used: $\lambda / 2 / \lambda / 4$ half-/quarter-waveplate, PBS—polarizing beamsplitter, DM-dichroic mirror, PDphotodiode, $\mathrm{MM}$-mode-matching lenses, and $\mathrm{AM}$-amplitude modulation.

high-frequency (6 GHz bandwidth), fiber-coupled waveguide EOM (AdvR Inc. WPM-P48P48-AL0-488 nm). The lasers are split using a dichromatic mirror to send the $457 \mathrm{~nm}$ light through a 1:1 telescope where the first lens is on a translation stage before recombining with the $470 \mathrm{~nm}$ light. This setup allows independent modematching of each laser to the ULE cavity. Each laser beam had $75 \mu \mathrm{W}$ of power incident on the ULE cavity. A standard PoundDrever-Hall (PDH) setup is used to produce error signals for laser stabilization. The reflected light is split using a dichromatic mirror allowing the $457 \mathrm{~nm}$ light and $470 \mathrm{~nm}$ light to be independently detected and demodulated at $20 \mathrm{MHz}$ by commercial photodetector/demodulation units (Stable Laser Systems PDH-1000-20B) to produce the $\mathrm{PDH}$ error signals.

The outputs of two computer-controlled function generators (Stanford Research Systems RF signal generators SG384 and SG382) are combined using an RF splitter/combiner (MiniCircuits ZFSC2-5-S+) and amplified (MiniCircuits ZFL-2500-VH+) to drive the high-frequency EOM. The function generators are referenced to a global positioning system (GPS) atomic-clock signal (Stanford Research Systems FS740) to prevent phase and frequency drifts. When in pulsed mode, function generator A is pulse-modulated with the modulation trigger sent to function generator B. Function generator B is set up in "blank" mode, which effectively reverses the polarity of the external modulation input so that when the output of function generator $\mathrm{A}$ is on, that of function generator B is off.

\section{RESULTS AND DISCUSSION}

Figure 2 shows the transmission of the $457 \mathrm{~nm}$ light through the ULE cavity as a function of frequency for both triple frequency modulation and pulsed triple frequency modulation $\left(f_{\text {pulse }}=20 \mathrm{kHz}\right)$ for $\Omega_{A} / 2 \pi=200 \mathrm{MHz}$ and $\Omega_{B} / 2 \pi=300 \mathrm{MHz}$. When in pulsed mode, the extraneous sidebands discussed in Sec. II D are eliminated. The power in the remaining sidebands also increases. For demonstration purposes, Fig. 3(a) shows only the $457 \mathrm{~nm}$ error signal in pulsed 


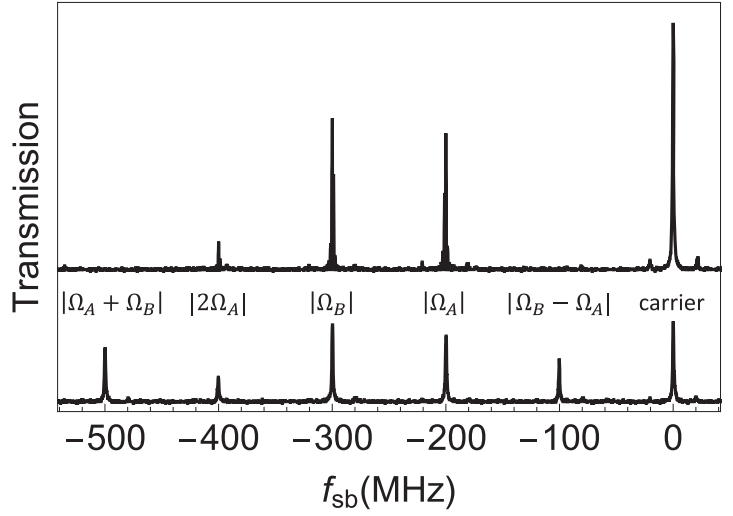

FIG. 2. Transmission of the $457 \mathrm{~nm}$ laser through the ULE cavity as a function of frequency with $\Omega_{A} / 2 \pi=200 \mathrm{MHz}$ and $\Omega_{B} / 2 \pi=300 \mathrm{MHz}$. $0 \mathrm{MHz}$ references to the carrier frequency. Bottom: triple frequency modulation. Top: pulsed triple frequency modulation. A vertical offset was added to the pulsed triple frequency modulation data to make it easier to view. Between the two plots are labels to indicate which frequency terms are produced from the modulation frequencies, $\Omega_{A}$ and $\Omega_{B}$. The $20 \mathrm{MHz}$ sidebands on each of these terms are present, but not always visible. For visual purposes, we only show the negative sidebands; the positive sidebands (not shown) are symmetric about the carrier.
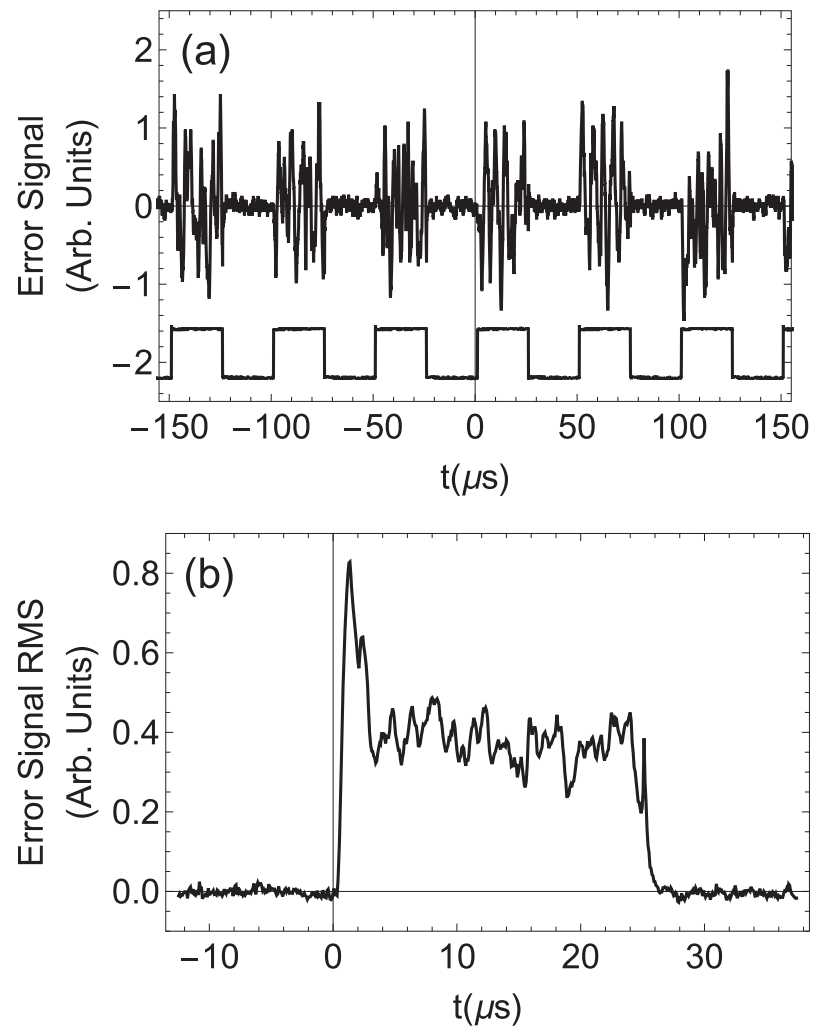

FIG. 3. (a) The locked ECDL error signal as a function of time with $f_{\text {pulse }}=20 \mathrm{kHz}$. Also shown is the modulation trigger. (b) The root mean square of 32 consecutive pulses shown in (a). The initial feature is the lock recapturing the laser due to drift when the pulse is off. mode after stabilization. Stabilization for the Toptica laser is done using Toptica's digital proportional-integral-derivative (PID) controller (DigiLock 110) with both low-frequency (to the piezo-electric transducer) and high-frequency (to the diode current) feedback. Figure 3(b) shows the root-mean square of the locked error signal for 32 consecutive pulses. At the beginning of the "on" pulse, the laser is recaptured by the lock electronics to be once again resonant with the ULE cavity mode. In general, the longer the pulse is off, the further the laser will drift from the resonant frequency and the larger this initial feature. The function generators allow pulsing with frequencies as high as $1 \mathrm{MHz}$. We successfully demonstrated this locking technique on the Toptica laser with pulse frequencies ranging from $1 \mathrm{MHz}$ to as low as $500 \mathrm{~Hz}$. However, if the laser drifts beyond the capture range of the error signal while its function generator is off, then stabilization will be lost. For many systems, it is advantageous to have a pulse frequency that is much larger than the bandwidth of the system. In this case, the chopped error signal can be averaged out. This illustrative example is specific to this ECDL laser and the bandwidth associated with this system. A different system, for example, our Ti:Sapphire laser with much smaller bandwidth, will have a different range of suitable pulse frequencies.

Residual amplitude modulation (RAM) is also important to consider and minimize in PDH stabilization. ${ }^{13,14}$ While minimizing RAM is often necessary, it is not feasible to completely remove it. In an ordinary PDH lock, the main issue arising from fluctuating RAM is drift in the error signal offset, leading to a drift in the locked laser frequency. When RAM is present in pulsed triple frequency modulation, there is an initial effect (see Fig. 4). While

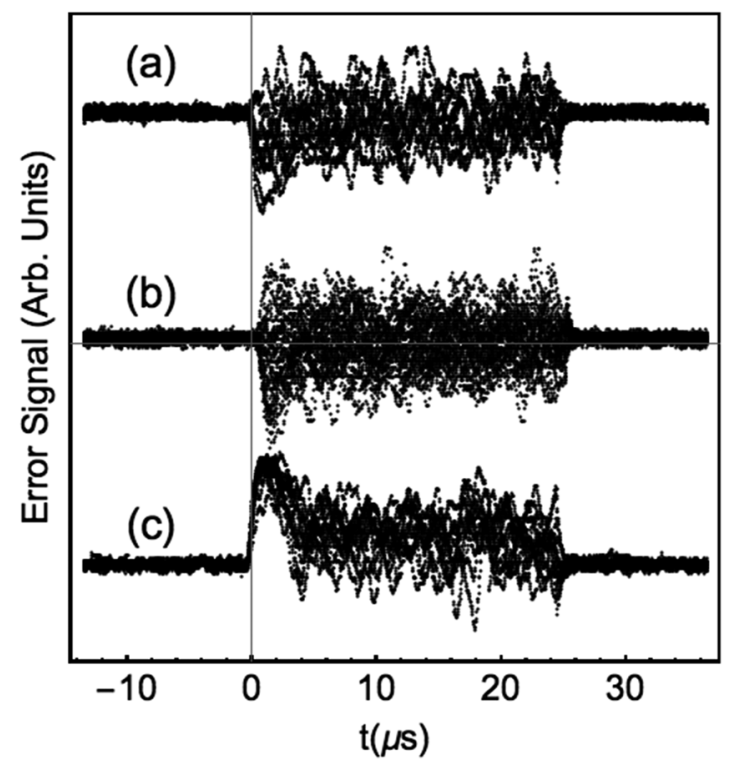

FIG. 4. The effects of residual amplitude modulation for a (a) negative offset, (b) negligible offset, and (c) positive offset. For these data, the positive offset is $\sim 3 x$ larger than the negative offset to display the effects of small vs large RAM. Each plot is 12 on/off sequences plotted simultaneously. 
the locking is "off," the voltage signal is at zero before locking to a non-zero voltage resulting from RAM. The result is that the initial feature from recapture is exacerbated. Figure 4 shows the effect of RAM when there is a negative offset (a), no offset (b), and a positive offset (c). For this figure, 32 locked error signals are plotted on top of one another to visually demonstrate the effects of RAM. Once recaptured, the rms value of the locked error signal is similar to that of the locked error signal when not in pulsed mode. However, a reoptimization of the lock parameters may be needed since the power in the sidebands is different in pulsed vs non-pulsed mode and lock bandwidth affects the recapture rate. Figure 5 shows both lasers locked to the ULE cavity in pulsed mode $\left(f_{\text {pulse }}=20\right.$ $\mathrm{kHz}$ ) and demonstrates the difference between a lower-bandwidth and a higher-bandwidth lock. For these data, the Ti-Sapphire laser is stabilized by using a commercial PID controller (Vescent Photonics D2-125) using only low-frequency (piezo-electric) feedback with an estimated lock bandwidth of about $300 \mathrm{~Hz}$, vs about $10 \mathrm{kHz}$ for the Toptica lock. When the trigger signal is high or the error signal for the Toptica laser is actively providing feedback, the ECDL servo bandwidth is large enough to visually see recapture and stabilization. When the trigger signal is low or the Ti-Sapphire laser error signal is actively providing feedback, the Ti-Sapphire servo bandwidth is smaller than the chopping frequency and stabilization is not evident on small time scales. Looking at a larger time scale for the low bandwidth lock will show oscillations around $0 \mathrm{~V}$ as expected for a stabilized laser. While Fig. 5 demonstrates the differences between two lasers with response times faster and slower than the pulse period $\left(T_{\text {pulse }}=1 / f_{\text {pulse }}\right)$, it is often desirable to have the pulse frequency much larger than the bandwidth: in this scenario, the pulse is effectively integrated out.

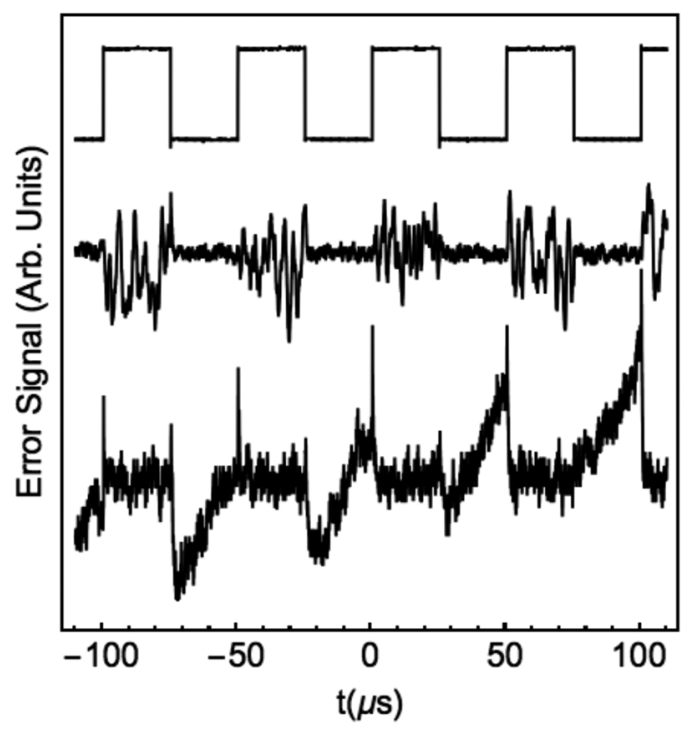

FIG. 5. The error signals when both lasers are locked. Top: the trigger signal that determines which servo is active. High is when the Toptica is "on" (locked to a sideband at $\Omega_{A} / 2 \pi=200 \mathrm{MHz}$ ), and low is when the Ti:Sapphire is "on" (locked to a sideband at $\Omega_{B} / 2 \pi=300 \mathrm{MHz}$ ). The middle plot is the error signal for the Toptica laser, and the bottom plot is the error signal for the Ti-Sapphire laser.
There are some limitations on the current setup that may be improved. As an example, consider scanning laser 1 with laser 2 fixed. Since the sidebands meant for laser 2 are still present on laser 1 , the scan range of laser 1 is limited by the spurious sidebands. For example, assuming a free spectral range of $1500 \mathrm{MHz}$, if laser 2 is stabilized to a negative sideband at $\Omega_{B} / 2 \pi=300 \mathrm{MHz}$, laser 1 can be scanned from $f_{E O M} \approx 20 \mathrm{MHz}$ to $280 \mathrm{MHz}$ (before interfering with the error signal at $300 \mathrm{MHz}$ ), from $\approx 320 \mathrm{MHz}$ to 730 $\mathrm{MHz}$ (before interfering with the positive sideband error signal at $750 \mathrm{MHz}$ ), from $\approx 770 \mathrm{MHz}$ to $1180 \mathrm{MHz}$ (before interfering with the positive $300 \mathrm{MHz}$ error signal that would appear to be at 1200 $\mathrm{MHz}$ ), and from $\approx 1220 \mathrm{MHz}$ to $1480 \mathrm{MHz}$ (before interfering with the positive sideband error signal and the carrier error signal that are both at $1500 \mathrm{MHz}$ ). To resolve this issue, we suggest adding an RF on/off switch before each of the lock electronics that is triggered by the corresponding function generator pulse trigger. This would prevent the spurious sidebands from creating an error signal that would disrupt the lock and effectively turn pulsed triple frequency modulation into independent dual frequency modulation for each laser.

\section{CONCLUSION}

In this paper, we presented a new technique to both stabilize and control the frequency of two lasers using a single optical cavity via simultaneous offset sideband stabilization with removal of error signals that could interfere with stabilization. When scanning the frequency of one of the lasers, undesirable error signals can interfere with the frequency of the locked lasers causing shifts in the lock points or loss of lock. Pulsed triple frequency modulation removes a large number of error signals so that the scan range of each laser is increased. This also has the advantage of reducing power loss to spurious sidebands. In general, the extent to which triple frequency modulation will increase the scan range depends on the experimental setup. However, it is possible to utilize an RF switch (as described in Sec. IV) so that the range would be half the free spectral range of the stabilization cavity, which is equivalent to the maximum dual frequency modulation scan range.

\section{ACKNOWLEDGMENTS}

This work was supported by the National Science Foundation through Grant Nos. PHY-1428112 and PHY-1555232.

\section{DATA AVAILABILITY}

The data that support the findings of this study are available from the corresponding author upon reasonable request.

\section{REFERENCES}

${ }^{1}$ K. L. Corwin, Z.-T. Lu, C. F. Hand, R. J. Epstein, and C. E. Wieman, Appl. Opt. 37, 3295 (1998).

${ }^{2}$ G. Jundt, G. T. Purves, C. S. Adams, and I. G. Hughes, Eur. Phys. J. D 27, 273 (2003).

${ }^{3}$ C. Wieman and T. W. Hänsch, Phys. Rev. Lett. 36, 1170 (1976).

${ }^{4}$ G. C. Bjorklund, Opt. Lett. 5, 15 (1980).

${ }^{\mathbf{5}}$ J. H. Shirley, Opt. Lett. 7, 537 (1982). 
${ }^{6}$ J. Zhang, D. Wei, C. Xie, and K. Peng, Opt. Express 11, 1338 (2003).

${ }^{7}$ R. W. P. Drever, J. L. Hall, F. V. Kowalski, J. Hough, G. M. Ford, A. J. Munley, and H. Ward, Appl. Phys. B 31, 97 (1983).

${ }^{8}$ B. G. Lindsay, K. A. Smith, and F. B. Dunning, Rev. Sci. Instrum. 62, 1656 (1991).

${ }^{9}$ J. I. Thorpe, K. Numata, and J. Livas, Opt. Express 16, 15980 (2008).

${ }^{10}$ E. C. Cook, A. D. Vira, C. Patterson, E. Livernois, and W. D. Williams, Phys. Rev. Lett. 121, 053001 (2018).
${ }^{11}$ E. C. Cook, A. D. Vira, and W. D. Williams, Phys. Rev. A 101, 042503 (2020).

${ }^{12}$ C. Patterson, A. D. Vira, M. T. Herd, W. B. Hawkins, and W. D. Williams, Rev. Sci. Instrum. 89, 033107 (2018).

${ }^{13}$ E. A. Whittaker, M. Gehrtz, and G. C. Bjorklund, J. Opt. Soc. Am. B 2, 1320 (1985).

${ }^{14}$ W. Zhang, M. J. Martin, C. Benko, J. L. Hall, J. Ye, C. Hagemann, T. Legero, U. Sterr, F. Riehle, G. D. Cole, and M. Aspelmeyer, Opt. Lett. 39, 1980 (2014). 